\title{
Towards next generation sequential and parallel SAT solvers
}

\author{
Norbert Manthey ${ }^{1}$
}

Published online: 10 September 2015

(C) Springer Science+Business Media New York 2015

\begin{abstract}
This thesis focuses on improving the SAT solving technology. The improvements focus on two major subjects: sequential SAT solving and parallel SAT solving.

To better understand sequential SAT algorithms, the abstract reduction system Generic CDCL is introduced. With Generic CDCL, the soundness of solving techniques can be modeled. Next, the conflict driven clause learning algorithm is extended with the three techniques local look-ahead, local probing and all UIP learning that allow more global reasoning during search. These techniques improve the performance of the sequential SAT solver Riss. Then, the formula simplification techniques bounded variable addition, covered literal elimination and an advanced cardinality constraint extraction are introduced. By using these techniques, the reasoning of the overall SAT solving tool chain becomes stronger than plain resolution. When using these three techniques in the formula simplification tool Coprocessor before using Riss to solve a formula, the performance can be improved further.
\end{abstract}

Due to the increasing number of cores in CPUs, the scalable parallel SAT solving approach iterative partitioning has been implemented in Pcasso for the multi-core architecture. Related work on parallel SAT solving has been studied to extract main ideas that can improve Pcasso. Besides parallel formula simplification with bounded variable elimination, the major extension is the extended clause sharing level based clause tagging, which builds the basis for conflict driven node killing. The latter allows to better identify unsatisfiable search space partitions. Another improvement is to combine scattering and look-ahead as a superior search space partitioning function. In combination with Coprocessor, the introduced extensions increase the performance of the parallel solver Pcasso. The implemented system turns out to be scalable for the multi-core architecture. Hence iterative partitioning is interesting for future parallel SAT solvers.

Norbert Manthey

norbert.manthey@tu-dresden.de

1 TU Dresden, Dresden, Germany 
The implemented solvers participated in international SAT competitions. In 2013 and 2014 Pcasso showed a good performance. Riss in combination with Coprocessor won several first, second and third prices, including two Kurt-Gödel-Medals. Hence, the introduced algorithms improved modern SAT solving technology.

School: TU Dresden

Supervisors:

Prof. Steffen Hölldober

Graduated: Monday, December 1, 2014

Link to full text: http://nbn-resolving.de/urn:nbn:de:bsz:14-qucosa-158672

\section{Notes:}

Nominated for ACP Doctoral Research Award, and GI-Dissertationspreis 2014 